\title{
Cannon waves in atrioventricular nodal re-entry tachycardia
}

\author{
Nagendra Boopathy Senguttuvan MD DM, Nilkanth Chandrakant Patil MD
}

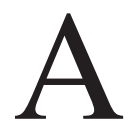

52-year-old woman presented to the emergency department with palpitations associated with uneasiness and nausea. She was a nonsmoker. She reported having one previous episode of palpitations, without precipitating factors, which had lasted for about 15 minutes and resolved spontaneously. Examination showed a regular heart rate of 185 beats/min with a blood pressure of 138/72 mm Hg. Very prominent regular jugular venous pulsations, suggestive of "cannon waves," were observed (Figure 1, Video 1). Cardiac examination was otherwise normal. Electrocardiography showed narrow complex regular tachycardia with a "pseudo $r$ " wave in V1 and "pseudo s" wave in the inferior leads, suggestive of atrioventricular nodal re-entry tachycardia (Appendix 1, available at www.cmaj.ca/lookup /suppl/doi:10.1503/cmaj.112155/-/DC1), which was treated with a rapid intravenous bolus of adenosine. The appearance of sinus rhythm after the administration of adenosine was simultaneously accompanied by the disappearance of cannon waves. She remained asymptomatic with metoprolol treatment during follow up.

Atrioventricular nodal re-entry tachycardia, which can occur at any age, is thought to be caused by dual atrioventricular nodal physiology with separate slow and fast pathways. Patients usually report palpitations, but they may also experience chest tightness, dyspnea, anxiety, presyncope or syncope, depending on the duration and rate of the tachycardia and the presence of structural heart disease. These episodes may be precipitated by smoking or using caffeine, drugs or alcohol. ${ }^{1}$ Although cannon waves are classically described with atrio ventricular dissociation ${ }^{2.3}$ seen with complete heart block or ventricular tachycardia, they may also be seen in atrioventricular nodal re-entry tachycardia, ${ }^{1}$ because of the near-simultaneous contraction of the ventricle and atrium. Cannon waves are regular in those with this condition, while they are usually irregular in ventricular tachycardia and complete heart block. ${ }^{1}$ Atrioventricular nodal re-entry tachycardia usually has a benign course. Although some episodes may be self-limiting, they frequently

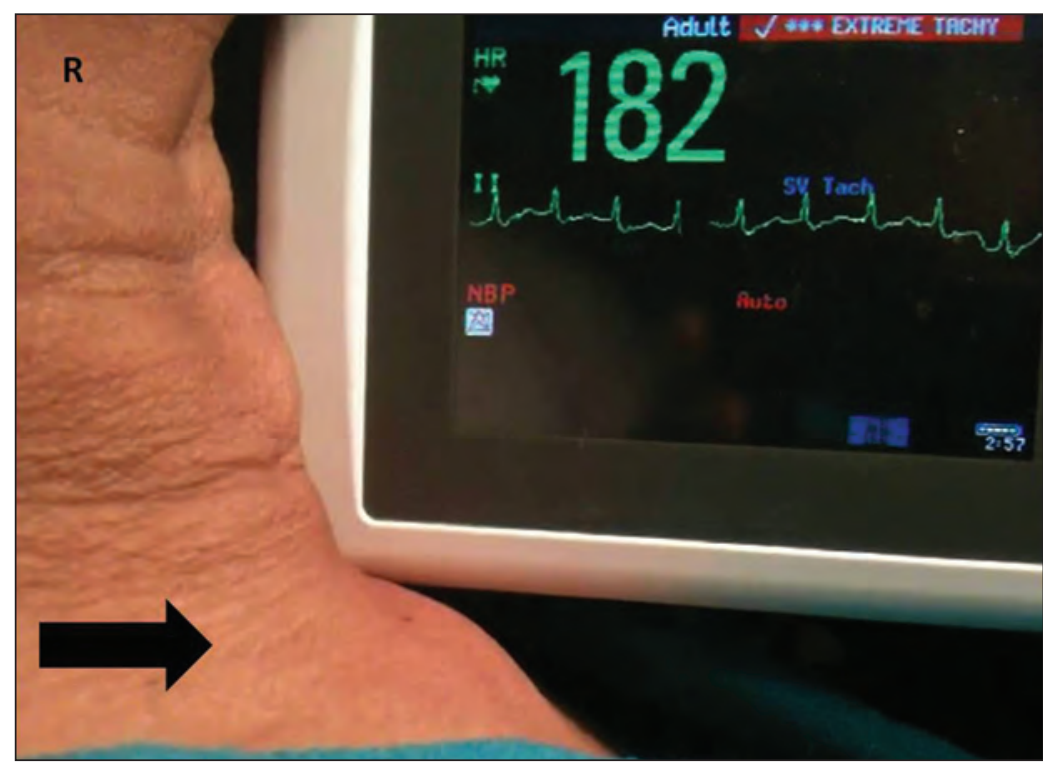

Figure 1: Cannon wave (arrow) in a 52-year-old woman with palpitations. require treatment. Guidelines recommend the use of vagal manoeuvres, adenosine, calcium channel blockers, $\beta$-blockers or digitalis during acute attacks. ${ }^{1}$ Refractory symptoms may be treated by radiofrequency ablation, which is effective in more than $96 \%$ of patients. ${ }^{1}$

\section{References}

1. Blomström-Lundqvist C, Scheinman MM, Aliot EM, et al. ACC/AHA/ESC guidelines for the management of patients with supraventricular arrhythmias - executive summary. A report of the American College of Cardiology/American Heart Association Task Force on Practice Guidelines and the European Society of Cardiology Committee for Practice Guidelines (writing committee to develop guidelines for the management of patients with supraventricular arrhythmias) developed in collaboration with NASPE-Heart Rhythm Society. J Am Coll Cardiol 2003;42: 1493-531.

2. Scherf D. "Cannon waves" in complete A-V block. Am Heart J 1971;82:577-8

3. Chen D. Pai PY. Cannon a wave. Circulation 2009;119:e381-3.
Competing interests: None declared.

This article has been peer reviewed.

Affiliation: From the Department of Cardiology, All India Institute of Medical Sciences, New Delhi, India.

Correspondence to: Nagendra Boopathy Senguttuvan, drsnboopathy @ gmail.com

CMAJ 2013. DOI:10.1503 /cmaj.112155
Please see the following video online: Cannon waves in atrioventricular nodal re-entry tachycardia www.cmaj.ca/lookup/suppl/doi:10.1503/cmaj.112155/-/DC1 